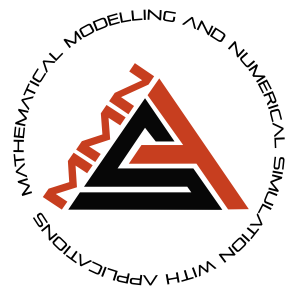

\title{
Construction of different types of traveling wave solutions of the relativistic wave equation associated with the Schrödinger equation
}

\author{
Asıf Yokus $1, *, \dagger$ \\ ${ }^{1}$ Department of Mathematics, Firat University, Elazig, 23200, Turkey \\ * Corresponding Author \\ †asfyokus@yahoo.com (Asıf Yokus)
}

\begin{abstract}
In this study, an alternative method has been applied to obtain the new wave solution of mathematical equations used in physics, engineering, and many applied sciences. We argue that this method can be used for some special nonlinear partial differential equations (NPDEs) in which the balancing methods are not integer. A number of new complex hyperbolic trigonometric traveling wave solutions have been successfully generated in the Eckhaus equation (EE) and nonlinear KleinGordon (nKG) equation models associated with the Schrödinger equation. The graphs representing the stationary wave are presented by giving specific values to the parameters contained in these solutions. Finally, some discussions about new complex solutions are given. It is discussed by giving physical meaning to the constants in traveling wave solutions, which are physically important as well as mathematically. These discussions are supported by three-dimensional simulation. In order to eliminate the complexity of the process and to save time, computer package programs have been utilized.
\end{abstract}

Key words: Eckhaus equation; nonlinear Klein Gordon equation; complex hyperbolic trigonometric travelling wave solutions; non-integer balancing term

AMS 2020 Classification: 35C07; 37M05; 83C15

\section{Introduction}

The proposed method has been shown to be an effective mathematical instrument to solve the nonlinear wave of equations in mathematics, physics, and engineering. So the discussion of NPDEs exact solutions in the nonlinear sciences is very important. Over the past few years, many researchers have used this beneficial method extensively, for example, the Jacobi elliptic expansion method [1], modified Kudryashov method [2, 3], the tanh method [4], sub-equation analytical method [5], the inverse scattering method [6], the first integral method[7, 8], the extended tanh-function method $[9,10]$, the Hirota's direct method [11], the auxiliary equation method [12], improved Bernoulli sub-equation function method [13], expansion method [14], $\left(G^{\prime} / G, 1 / G\right)$-expansion method [15, 16, 17], generalized exponential rational function method $[18,19,24]$, Sinh-Gordon function method [20], Sine-Gordon expansion method [21], Bernoulli sub-equation method [22], $\left(G^{\prime} / G\right)$-expansion method [23].

The equation of the Eckhaus is as follows [25]:

$$
i u_{t}+u_{x x}+2\left(|u|^{2}\right)_{x} u+|u|^{4} u=0,
$$

where $u=u(x, t)$ is a complex function. Eq. (1) is of the Schrödinger nonlinear type and recognizes a linearization of the Schrödinger linear equation that depends on free time. The EE has been found as a multi-scale asymptotic reduction of certain classes of NPDE. 
The EE was linearized by a variation of the dependent variable. Many scientists have worked with this equation for example, the first-integral method [25], weakly nonlinear effects [26], the Laplace transform [27], the EE was linearized by an appropriate change of variable [28], many of the properties of the EE were researched [29], the EE can be integrated by a change of dependent variable [30], the intent of this work is to discover exact solutions of the EE by expansion method.

In this work, we use the expansion method to contract several new cases with the exact solutions for some NPDEs such as the nKG equation, which in mathematical physics is very important and many researchers paid attention to the balance number, not integer. In this paper, we analyze the nKG equation [31] as follows:

$$
u_{t t}-\omega^{2} u_{x x}+\alpha u-\beta u^{3}+\gamma u^{5}=0
$$

where $\omega, \alpha, \beta$, and $\gamma$ are arbitrary constant. In many scientific applications, these equations play an important role, such as the quantum field theory [32], the solid state physics [33], the nKG equations, and found many types of exact traveling wave solutions including compact solutions, periodic solutions, soliton solution using the tanh-function method [34], generalized Kudryashov method [35], Homotopy Perturbation Method [36].

The nonlinear Klein-Godon equation, which is directly related to the Schrödinger equation, has become famous in the literature as the relativistic wave equation[37]. It is also one of the indispensable equations of relativistic quantum mechanics, which examines the behavior of particles exposed to high energy. Space and rocket industry, nuclear and medical waste treatment, earthquake, high energy, and plasma physics are the application areas.

\section{The methodology of the $\left(1 / G^{\prime}\right)$-expansion method}

In this segment, general realities of the $\left(1 / G^{\prime}\right)$-expansion method $[38,39,40]$ are displayed. To start with, we consider the general type of nonlinear PDE that depends on $t$ and $x$ variables.

$$
W\left(u, u_{t}, u_{x}, u_{x x}, \ldots\right)=0 \text {, }
$$

here is $u(x, t)$ a function that depends on $x$ and $t, \xi=k(x-2 a t)$ in the form of $u(x, t)=U e^{i(a x+b t)}$ where $k, a$ and $b$ are constants that are not zero. PDE referred to as Eq. (3), using this conversions

$$
Q\left(U, U^{\prime}, U^{\prime \prime}, \ldots\right)=0
$$

the ODE is shaped. On the other hand, the solution of the linear ODE is given below $G=G(\xi)$.

$$
G^{\prime \prime}+\lambda G^{\prime}+\mu=0
$$

The solution of nonlinear ODE given by Eq. (4) can be written as follows.

$$
u(\xi)=\sum_{i=1}^{m} a_{i}\left(\frac{1}{G^{\prime}}\right)^{m}
$$

here are $a_{1}, a_{2}, \ldots, \lambda, \mu$ constants and $m$ is the balancing term. The term balance is a fixed number obtained in any non-linear ODE between the highest order linear term and the highest order non - linear term. This number Eq. (6) is written in place and then the needful derivatives for the solution are obtained. In such derivatives, $G^{\prime \prime}=-\lambda G-\mu$ taken as $\left(1 / G^{\prime}\right)$ is a polynomial and homogeneous equation. Here $\left(1 / G^{\prime}\right)^{m}, m \in Q$ equals the coefficients of the terms to zero and a system of algebraic equations is built. This algebraic system of equations is calculated by using computer technology. These constants are written in place in Eq. (6). The $\xi$-linked solution must provide Eq. (4). After the necessary controls, the wave transformation is reversed and we reach the solution of Eq. (3).

\section{Application 1}

In this section, the solution given by Eq. (1) with $\left(1 / G^{\prime}\right)$-expansion method will be obtained. We can choose the following transformation of the wave for Eq. (1):

$$
u(x, t)=U(\xi) e^{i(a x+b t)}, \quad \xi=k(x-2 a t),
$$

under Eq. (1) wave transformation, Eq. (1) is converted to ODE as follows:

$$
k^{2} U^{\prime \prime}-\left(b+a^{2}\right) U+4 k U^{\prime} U^{2}+U^{5}=0 .
$$

In Eq. (8), the balancing constant between the highest order linear term $U^{\prime \prime}$ and the highest nonlinear term $U^{5}$ is that $m=\frac{1}{2}$ is not integer. The solution of the ODE obtained in the form of Eq. (8) can be given in the following way considering Eq. (6):

$$
U(\xi)=a_{1}\left(\frac{1}{G^{\prime}}\right)^{\frac{1}{2}}
$$


we take the 1st and 2nd derivatives of Eq. (9) and put them in Eq. (8), we get a polynomial with $\left(1 / G^{\prime}\right)^{\frac{r}{2}}, \quad r \in N$ variable. $\left(1 / G^{\prime}\right)^{\frac{r}{2}}$ the polynomial term coefficients equal to zero.

$$
\begin{array}{ll}
\left(\frac{1}{G^{\prime}}\right)^{\frac{1}{2}}: & -a^{2} a_{1}-b a_{1}+\frac{1}{4} k^{2} \lambda^{2} a_{1}=0, \\
\left(\frac{1}{G^{\prime}}\right)^{\frac{3}{2}}: & k^{2} \lambda \mu a_{1}+2 k \lambda a_{1}^{3}=0, \\
\left(\frac{1}{G^{\prime}}\right)^{\frac{5}{2}}: & \frac{3}{4} k^{2} \mu^{2} a_{1}+2 k \mu a_{1}^{3}+a_{1}^{5}=0 .
\end{array}
$$

The system of Eq. (10) can be solved with the help of a computer package program. The solutions obtained here are put into Eq. (9). Finally, the conversion is reversed and a new complex hyperbolic trigonometric travelling wave solution for Eq. (1) is obtained. The results are as follows:

$$
\begin{gathered}
a_{1}=\mp \frac{\mathrm{i} \sqrt{k} \sqrt{\mu}}{\sqrt{2}}, \quad \lambda=\mp \frac{2 \sqrt{a^{2}+b}}{k}, \\
u(x, t)=-\frac{\mathrm{ie}^{\mathrm{i}(a x+b t) \sqrt{k} \sqrt{\mu}}}{\sqrt{2}}\left(\frac{1}{\frac{k \mu}{2 \sqrt{a^{2}+b}}+A \cosh \left[2(x-2 a t) \sqrt{a^{2}+b}\right]+A \sinh \left[2(x-2 a t) \sqrt{a^{2}+b}\right]}\right)^{\frac{1}{2}},
\end{gathered}
$$
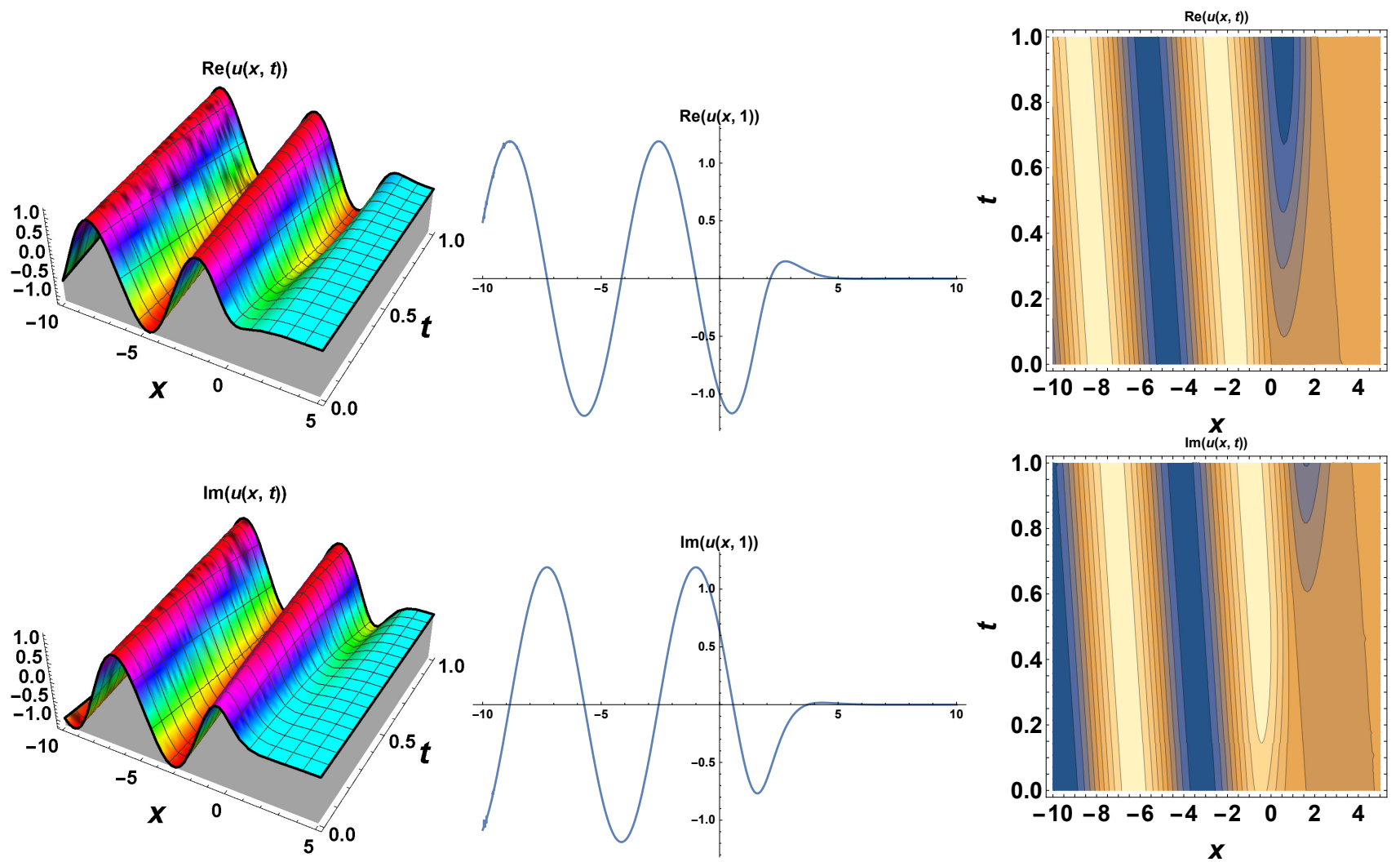

Figure 1. In terms of the Eq (11), for the constants $a=1, b=1, A=-5, k=-1, \mu=6$ the new complex hyperbolic trigonometric travelling wave solution of Eq. (1).

\section{Application 2}

In this section, we use the proposed method to solve the nKG equation. We can choose the following transformation of the wave for Eq. (2):

$$
u(x, t)=U(\xi), \quad \xi=x-V t
$$

where $V$ is constant. The travelling wave variable Eq. (12) allows us to convert Eq. (2) to the following ODE for $U=U(\xi)$ :

$$
\left(V^{2}-\omega^{2}\right) U^{\prime \prime}+\alpha U-\beta U^{3}+\gamma U^{5}=0
$$


In Eq. (13), the balancing constant between the highest order linear term $U^{\prime \prime}$ and the highest nonlinear term $U^{5}$ is that $m=\frac{1}{2}$ is not integer. The solution of the ODE obtained in the form of Eq. (13) can be given in the following way considering Eq. (6).

$$
U(\xi)=a_{1}\left(\frac{1}{G^{\prime}}\right)^{\frac{1}{2}}
$$

we take the 1st and 2nd derivatives of Eq. (14) and put them in Eq. (13), we get a polynomial with $\left(1 / G^{\prime}\right)^{\frac{r}{2}}, \quad r \in N$ variable. $\left(1 / G^{\prime}\right)^{\frac{r}{2}}$ the polynomial term coefficients equal to zero.

$$
\begin{array}{ll}
\left(\frac{1}{G^{\prime}}\right)^{\frac{1}{2}}: & \alpha a_{1}-\frac{1}{4} \omega^{2} \lambda^{2} a_{1}+\frac{1}{4} v^{2} \lambda^{2} a_{1}=0, \\
\left(\frac{1}{G^{\prime}}\right)^{\frac{3}{2}}: & -\omega^{2} \lambda \mu a_{1}+v^{2} \lambda \mu a_{1}-\beta a_{1}^{3}=0, \\
\left(\frac{1}{G^{\prime}}\right)^{\frac{5}{2}}: & -\frac{3}{4} \omega^{2} \mu^{2} a_{1}+\frac{3}{4} \nu^{2} \mu^{2} a_{1}+\gamma a_{1}^{5}=0,
\end{array}
$$

The system of Eq. (15) can be solved with the help of a computer package program. The solutions obtained here are put into Eq. (14). Finally, the conversion is reversed and a new complex hyperbolic type trigonometric travelling wave solution to Eq. (2) is obtained. The results are as follows:

$$
\begin{gathered}
a_{1}=\mp \frac{2 \mathrm{i} \sqrt{\alpha} \sqrt{\mu}}{\sqrt{\beta} \sqrt{\lambda}}, \quad \gamma=\frac{3 \beta^{2}}{16 \alpha}, \quad v=\mp \frac{\sqrt{-4 \alpha+\omega^{2} \lambda^{2}}}{\lambda}, \\
u(x, t)=-\frac{2 \mathrm{i} \sqrt{\alpha} \sqrt{\mu}}{\sqrt{\beta} \sqrt{\lambda}\left[-\frac{\mu}{\lambda}+A \cosh \left[\lambda\left(x+\frac{t \sqrt{-4 \alpha+\omega^{2} \lambda^{2}}}{\lambda}\right)\right]-A \sinh \left[\lambda\left(x+\frac{t \sqrt{-4 \alpha+\omega^{2} \lambda^{2}}}{\lambda}\right)\right]\right]^{\frac{1}{2}}} .
\end{gathered}
$$

$\operatorname{Re}(u(x, t))$

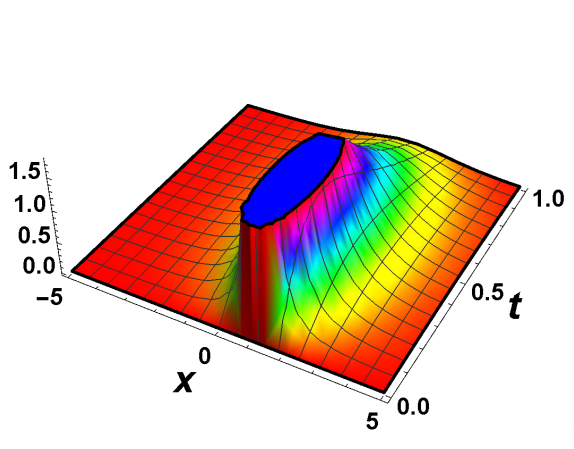

$\operatorname{lm}(u(x, t))$

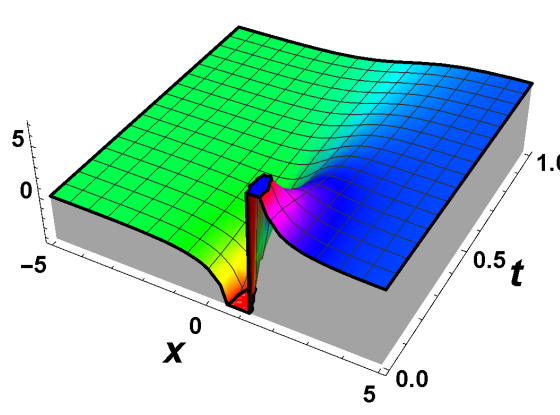

Figure 2. In terms of Eq. (16), for the constants $\alpha=2, \beta=1, A=3, k=2.5, \mu=1, \lambda=1, \omega=0.001$ the new complex hyperbolic trigonometric travelling wave solution of Eq. (2).

\section{Results and discussions}

In this study, we have introduced a new method for a new complex hyperbolic trigonometric travelling wave solution of partial differential equations that are not integer in the balancing term. We have implemented the application here for the balancing term $m=\frac{1}{2}$. In next studies, both $m<0$ and $m \neq \frac{1}{2}$ can be used for balancing terms that $m$ is not integer. For Eq. (1), the complex hyperbolic trigonometric travelling wave solution presented in the form of Eq. (11), and for Eq. (2), the complex hyperbolic trigonometric travelling wave solution presented in the form of Eq. (16) is new and the solutions provide Eq. (1) and Eq. (2). 
By giving special values to the parameters in these solutions, we can present the three-dimensional graphics representing the constant wave as follows.

The traveling wave solutions obtained in this study are valuable physically as well as mathematically. In order to understand its physical value, the constants in the obtained traveling wave solutions must be given physical meaning. In physics, the mathematical model representing the assumption that the envelope of a forward-moving wave pulse changes slowly in time and space compared to a period or wavelength can be represented by Eq. (7) [41]. In Eq. (7), $\xi=k(x-2 a t)$ is the variable of the traveling wave. $U(\xi)$ represents an amplitude of the traveling wave solution. In addition, $k a$ and $a$ represent the velocity and frequency of the traveling wave, respectively, which is physically prominent in this study and whose different values will be analyzed for the behavior of the traveling wave. The $a$ parameter is directly related to both the speed of the wave and the length of the wave. So let us analyze the behavior simulation of the traveling wave solution for different values of $a$ in Eq. (11) traveling wave solution produced by the $\left(1 / G^{\prime}\right)$-expansion method for Eq. (1).

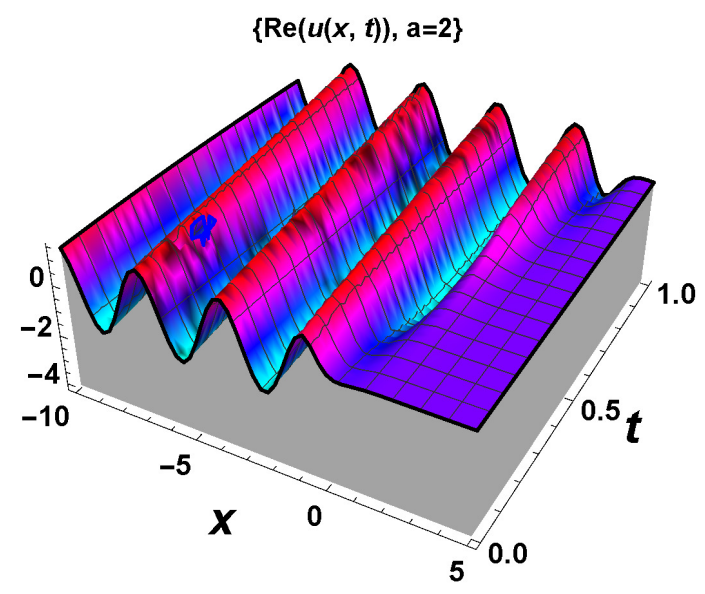

$\{\operatorname{Re}(u(x, t)), \mathrm{a}=4\}$

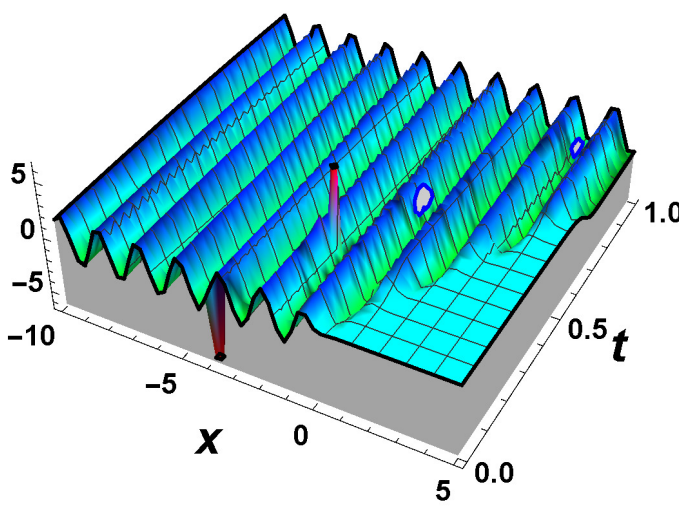

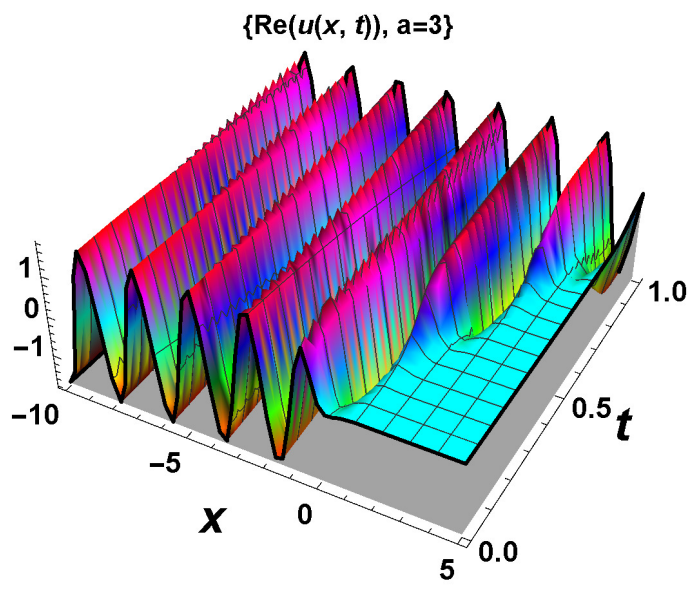

$\{\operatorname{Re}(u(x, t)), \mathrm{a}=5\}$

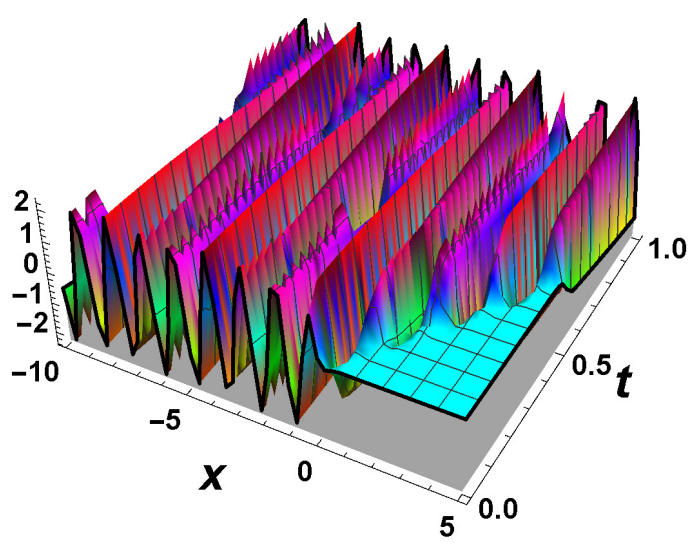

Figure 3. In terms real part of Eq. (11), for the constants $b=1, A=-5, k=-1, \mu=1$ the new complex hyperbolic trigonometric travelling wave solution of Eq. (1). 

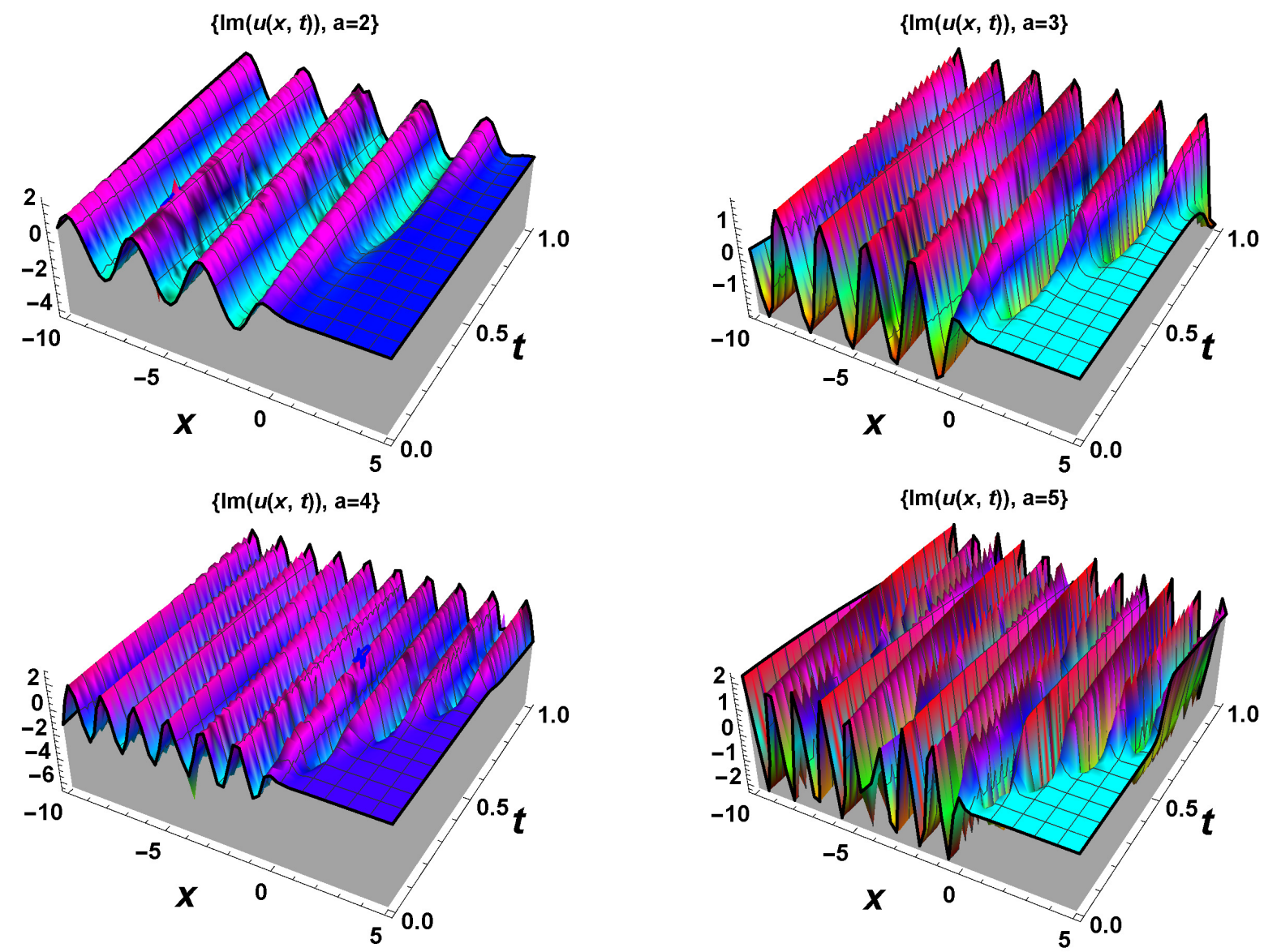

Figure 4. In terms imaginary part of Eq. (11), for the constants $b=1, A=-5, k=-1, \mu=1$ the new complex hyperbolic trigonometric travelling wave solution of Eq. (1).

Physically, we can observe in Figs. 3 and 4 that the parameter $a$ is related to both the velocity and the frequency of the traveling wave. As the frequency increases, the wavelength of the traveling wave decreases while its speed increases. When $a=$, the traveling wave exhibits triangular wave behavior. The frequency of the traveling wave is directly related to the wave number. As the frequency increases, the number of waves increases. We can support the validity of this discussion with Figs. 3 and 4 . In addition, the interpretations are similar as it exhibits similar behaviors for the real and imaginary parts.

The Klein-Gordon equation is related to the Schrödinger equation and is known as the relativistic wave equation. In physics, relativistic quantum mechanics studies the behavior of particles exposed to high energy [37]. We think that the Klein-Gordon equation, whose traveling wave solution is produced in this study, can be a mathematical model of a high-energy seismic wave. Traveling wave solutions, which play an important role in the transfer of energy from one point to another, may lead to different discussions about earthquakes in the future.

\section{Conclusions}

In this paper, we explored a new application $\left(1 / G^{\prime}\right)$-expansion method, we obtained exact solutions of the EE and nKG equation, and obtained new types of complex hyperbolic trigonometric travelling wave solution for the EE and nKG equation. Many scientists were less interested, when the balancing term used in the expansion methods was non-integer or negative. In order to increase this interest, the method can be applied for non-integer balancing terms of partial differential equations. This paper presented wider applicability using the $\left(1 / G^{\prime}\right)$-expansion method to handle nonlinear evolutionary equations. The parameters in the obtained traveling wave solution were given physical meaning. A three-dimensional simulation of the wave behavior is constructed for different values of the $a$ parameter, which affects the frequency and speed of the traveling wave. The dynamics of the wave supported by simulation are discussed. Results show that this method was effective. The new wave solution obtained in this paper can present different perspectives on future researches. 


\section{Declarations}

\section{Consent for publication}

Not applicable.

\section{Conflicts of interest}

The author declares that he has no known competing financial interests or personal relationships that could have appeared to influence the work reported in this paper.

\section{Funding}

The author declares that there is no funding source for the reported research.

\section{Author's contributions}

The research was carried out by the author and he accepts that the contributions and responsibilities belong to the author.

\section{Acknowledgements}

The author would like to thank Firat University for their unwavering support in the conduct of the research.

\section{References}

[1] Alquran, M., Jarrah, A., and Krishnan, E. Solitary Wave Solutions of the Phi-Four Equation and the Breaking Soliton System by Means of Jacobi Elliptic Sine-Cosine Expansion Method. Nonlinear Dynamics and Systems Theory, 18(3), 233-240, (2018).

[2] Durur, H., and Yokuş, A. Discussions on diffraction and the dispersion for traveling wave solutions of the (2+1)-dimensional paraxial wave equation. Mathematical Sciences, 1-11, (2021) https://link.springer.com/article/10.1007/s40096-021-00419-z.

[3] Yokuş, A., Durur, H., and Duran, S. Simulation and refraction event of complex hyperbolic type solitary wave in plasma and optical fiber for the perturbed Chen-Lee-Liu equation. Optical and Quantum Electronics, 53(402), (2021) https://doi.org/10.1007/s11082-021-03036-1.

[4] Malfliet, W. and Hereman, W. The tanh method: II. Perturbation technique for conservative systems. Physica Scripta, 54(6), 569, (1996).

[5] Duran, S., and Karabulut, B. Nematicons in liquid crystals with Kerr Law by sub-equation method. Alexandria Engineering Journal, (2021) https://doi.org/10.1016/j.aej.2021.06.077.

[6] Caudrelier, V.. Interplay between the Inverse Scattering Method and Fokas's Unified Transform with an Application. Studies in Applied Mathematics, 140(1), 3-26, (2018).

[7] Zhang, Q., Xiong, M., and Chen, L. The First Integral Method for Solving Exact Solutions of Two Higher Order Nonlinear Schrödinger Equations, Journal of Advances in Applied Mathematics, 4(1), (2019).

[8] Feng, Z. and Wang, X. The first integral method to the two-dimensional Burgers-Korteweg-de Vries equation. Physics Letters A, 308(2-3), 173-178, (2003).

[9] Fan, E. Extended tanh-function method and its applications to nonlinear equations. Physics Letters A, 277(4-5), 212-218, (2000).

[10] Tariq, H. et al. New travelling wave analytic and residual power series solutions of conformable Caudrey-Dodd-GibbonSawada-Kotera equation. Results in Physics, 104591, (2021).

[11] Wazwaz, A.-M. The Hirota's direct method and the tanh-coth method for multiple-soliton solutions of the Sawada-Kotera-Ito seventh-order equation. Applied Mathematics and Computation, 199(1), 133-138, (2008).

[12] Akbulut, A. and Kaplan, M. Auxiliary equation method for time-fractional differential equations with conformable derivative. Computers \& Mathematics with Applications, 75(3), 876-882, (2018).

[13] Baskonus, H.M. and Bulut, H. On the complex structures of Kundu-Eckhaus equation via improved Bernoulli sub-equation function method, Waves in Random and Complex Media, 25(4), 720-728, (2015).

[14] Wang, M., Li, X., and Zhang, J. The ( $\left.G^{\prime} / G\right)$-expansion method and travelling wave solutions of nonlinear evolution equations in mathematical physics. Physics Letters A, 372(4), 417-423, (2018).

[15] Duran, S. Extractions of travelling wave solutions of $(2+1)$-dimensional Boiti-Leon-Pempinelli system via $\left(G^{\prime} / G, 1 / G\right)$-expansion method. Optical and Quantum Electronics, 53(6), 1-12, (2021).

[16] Duran, S. Solitary Wave Solutions of the Coupled Konno-Oono Equation by using the Functional Variable Method and the Two Variables $\left(G^{\prime} / G, 1 / G\right)$-Expansion Method. Adiyaman University Journal of Science, 10(2), 585-594, (2020).

[17] Yokuş, A., and Durur, H. $\left(G^{\prime} / G, 1 / G\right)$-expansion method for analytical solutions of Jimbo-Miwa equation. Cumhuriyet Science Journal, 42(1), 88-98, (2021).

[18] Durur, H. Energy-carrying wave simulation of the Lonngren-wave equation in semiconductor materials. International Journal of Modern Physics B, 2150213, (2021).

[19] Ali, K.K., Yilmazer, R., Bulut, H., Aktürk, T., and Osman, M.S. Abundant exact solutions to the strain wave equation in micro-structured solids. Modern Physics Letters B, 2150439, (2021).

[20] Yokuş, A., Durur, H., Nofal, T.A., Abu-Zinadah, H., Tuz, M., and Ahmad, H. Study on the applications of two analytical 
methods for the construction of traveling wave solutions of the modified equal width equation. Open Physics, 18(1), 1003-1010, (2020).

[21] Ali, K K., Yilmazer, R., Baskonus, H.M., and Bulut, H. New wave behaviors and stability analysis of the Gilson-Pickering equation in plasma physics. Indian Journal of Physics, 95(5), 1003-1008, (2021).

[22] Ali, K.K., Yilmazer, R., Baskonus, H.M., and Bulut, H. Modulation instability analysis and analytical solutions to the system of equations for the ion sound and Langmuir waves. Physica Scripta, 95(6), 065602, (2020).

[23] Akinyemi, L., Şenol, M., Rezazadeh, H., Ahmad, H., and Wang, H. Abundant optical soliton solutions for an integrable (2+ 1)-dimensional nonlinear conformable Schrödinger system. Results in Physics, 25, 104177, (2021).

[24] Duran, S. Breaking theory of solitary waves for the Riemann wave equation in fluid dynamics. International Journal of Modern Physics B, 2150130, (2021).

[25] Taghizadeh, N., Mirzazadeh, M., and Tascan, F., The first-integral method applied to the Eckhaus equation, Applied Mathematics Letters, 25(5), 798-802, (2012).

[26] Calogero, F. and Eckhaus, W. Nonlinear evolution equations, rescalings, model PDEs and their integrability: II. Inverse Problems, 4(1), 11, (1988).

[27] Calogero, F. and De Lillo, S. Cauchy problems on the semiline and on a finite interval for the Eckhaus equation. Inverse Problems, 4(4), L33, (1988).

[28] Calogero, F. The evolution partial differential equation ut $=u x x x+3(u x x u 2+3 u 2 x u)+3 u x u$ 4. Journal of mathematical physics, 28(3), 538-555, (1987).

[29] Calogero, F. and De Lillo, S. The Eckhaus PDE $i \psi t+\psi x x+2\left(|\psi|^{2}\right) x \psi+|\psi| 4 \psi=0$. Inverse Problems, 3(4), 633, (1987).

[30] Calogero, F. and De Lillo, S. The Eckhaus equation in an external potential. Journal of Physics A: Mathematical and General, 25(7), L287, (1992).

[31] Zayed, E.M. and Gepreel, K.A. The modified $\left(G^{\prime} / G\right)$-expansion method and its applications to construct exact solutions for nonlinear PDEs. Wseas transactions on mathematics, 10(8), 270-278, (2011).

[32] Peskin, M.E. An introduction to quantum field theory, CRC Press, (2018).

[33] Khalatnikov, I.M. An introduction to the theory of superfluidity, CRC Press, (2018).

[34] Wazwaz, A.-M. Compactons, solitons and periodic solutions for some forms of nonlinear Klein-Gordon equations. Chaos, Solitons \& Fractals, 2(4), 1005-1013, (2006).

[35] Baskonus, H.M. and Bulut, H. New hyperbolic function solutions for some nonlinear partial differential equation arising in mathematical physics, Entropy, 17(6), 4255-4270, (2015).

[36] Bulut, H. and Başkonus, H.M., On The Geometric Interpretations of The Klein-Gordon Equation And Solution of The Equation by Homotopy Perturbation Method. Applications \& Applied Mathematics, 7(2), (2012).

[37] Orion, T. Klein-Gordon equation. Encyclopedia of Mathematics, (2012). https://encyclopediaofmath.org/wiki/Klein_Gordonequation.

[38] Yokus, A. Comparison of Caputo and conformable derivatives for time-fractional Korteweg-de Vries equation via the finite difference method. International Journal of Modern Physics B, 32(29), 1850365, (2018).

[39] Yokus, A. and Kaya, D. Numerical and exact solutions for time fractional Burgers' equation. Journal of Nonlinear Sciences and Applications, 10(7), 3419-3428, (2017).

[40] Yokus, A. and Kaya, D. Conservation laws and a new expansion method for sixth order Boussinesq equation. in AIP Conference Proceedings, AIP Publishing, (2015).

[41] Arecchi, F. and Bonifacio, R. Theory of optical maser amplifiers. IEEE Journal of Quantum Electronics, 1(4), 169-178, (1965).

Mathematical Modelling and Numerical Simulation with Applications (MMNSA) (http://www.mmnsa.org)

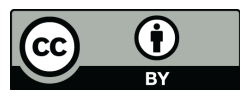

Copyright: ( $) 2021$ by the authors. This work is licensed under a Creative Commons Attribution 4.0 (CC BY) International License. The authors retain ownership of the copyright for their article, but they allow anyone to download, reuse, reprint, modify, distribute, and/or copy articles in MMNSA, so long as the original authors and source are credited. To see the complete license contents, please visit (http://creativecommons.org/licenses/by/4.0/). 